ARTICLE

https://doi.org/10.1038/s41467-019-09926-z

\title{
Individual variability in behavior and functional networks predicts vulnerability using an animal model of PTSD
}

David Dopfel (10) 1,5, Pablo D. Perez 1,5, Alexander Verbitsky², Hector Bravo-Rivera ${ }^{3}$, Yuncong Ma1, Gregory J. Quirk ${ }^{3,4} \&$ Nanyin Zhang (1) ${ }^{1}$

Only a minority of individuals experiencing trauma subsequently develop post-traumatic stress disorder (PTSD). However, whether differences in vulnerability to PTSD result from a predisposition or trauma exposure remains unclear. A major challenge in differentiating these possibilities is that clinical studies focus on individuals already exposed to trauma without pre-trauma conditions. Here, using the predator scent model of PTSD in rats and a longitudinal design, we measure pre-trauma brain-wide neural circuit functional connectivity, behavioral and corticosterone responses to trauma exposure, and post-trauma anxiety. Freezing during predator scent exposure correlates with functional connectivity in a set of neural circuits, indicating pre-existing circuit function can predispose animals to differential fearful responses to threats. Counterintuitively, rats with lower freezing show more avoidance of the predator scent, a prolonged corticosterone response, and higher anxiety long after exposure. This study provides a framework of pre-existing circuit function that determines threat responses, which might directly relate to PTSD-like behaviors.

\footnotetext{
${ }^{1}$ Department of Biomedical Engineering, Pennsylvania State University, University Park, PA 16802, USA. ${ }^{2}$ Department of Engineering Science and Mechanics, Pennsylvania State University, University Park, PA 16802, USA. ${ }^{3}$ Department of Anatomy \& Neurobiology, University of Puerto Rico School of Medicine, San Juan 00936, Puerto Rico. ${ }^{4}$ Department of Psychiatry, University of Puerto Rico School of Medicine, San Juan 00936, Puerto Rico. ${ }^{5}$ These authors contributed equally: David Dopfel, Pablo D. Perez. Correspondence and requests for materials should be addressed to N.Z. (email: nuz2@psu.edu)
} 
$\mathrm{M}$ aladaptive responses to trauma may lead to chronic stress disorders like post traumatic stress disorder (PTSD). While trauma exposure is a feature of PTSD, it is not an unequivocal predictor of the disorder. In fact, only a minority of individuals who experience traumatic event(s) subsequently develop PTSD. However, mechanisms underlying this individual difference in vulnerability remain unclear.

It has been suggested that vulnerability to PTSD may result from individual differences in early life events, pre-existing conditions, or responses to trauma exposure ${ }^{1-3}$. However, mixed results have been reported. For instance, reduced hippocampal volume has long been considered a marker of PTSD. Nevertheless, some studies suggest that PTSD-related decreases in hippocampal volume might be a consequence of traumatic events and PTSD development, whereas other studies showed that PTSD patients' twins who did not have a trauma experience also had smaller hippocampal volumes, suggesting that reduced hippocampal volume may predispose individuals to developing PTSD ${ }^{4-}$ 7. These conflicting results are also reported in studies of cortisol levels and brain activity in relation to $\mathrm{PTSD}^{8-10}$, which together contribute to our elusive understanding of individual vulnerability to PTSD.

A major obstacle of investigating risk factors of PTSD is rooted in the difficulty of longitudinally monitoring PTSD development pre- through post trauma in humans with well-controlled traumatic events. Studies in humans focus on populations already exposed to a variety of uncontrolled traumatic events ${ }^{11}$. Such barriers can be overcome in experimental animals, for which a longitudinal design with controlled traumatic stressors can be conveniently applied. Among many stress paradigms that have been used to produce rat models of PTSD, predator scent exposure in an inescapable environment is a commonly used psychological stressor ${ }^{12}$. This method mimics a life-threatening situation that is ecologically relevant and physically innocuous, preventing confounding effects of inflammation or pain due to injury. Recent work with this model has found that PTSD-like behaviors are exhibited following exposure to predator scent ${ }^{13}$. Importantly, Cohen et al. distinguished the responses of mal- vs. well-adapted rats, showing a $25-30 \%$ prevalence of PTSD-like behavior in rats exposed to trauma ${ }^{14}$. This work highlights the potential for this animal model to investigate relevant individual variability in PTSD-like responses to trauma. Missing from previous studies, however, is information about pre-existing conditions of animals that might predict their immediate and longterm responses to trauma.

Additionally, little is known about whether and how specific defense responses to a threat correlate with susceptibility to maladaptation. Exposure to a stressor elicits different fear responses depending on the perceived proximity and intensity of the threat. Moderate stressors produce passive freezing, while imminent predation threats elicit a fight or flight response ${ }^{15,16}$. However, whether behavioral responses to predator scent is a useful indicator of subsequent development of PTSD is unknown.

Here, we employ a longitudinal approach to studying individual variability of behavioral and functional outcomes of the predator scent animal model of PTSD. Resting-state functional magnetic resonance imaging (rsfMRI) data are collected from awake rodents before trauma exposure to record pre-existing neural circuit function using the awake rat rsfMRI approach established in our lab ${ }^{17-20}$. Awake rat rsfMRI avoids confounding factors from anesthetics and permits correlation with behavior ${ }^{21-}$ 23. We find that individual differences in behavior and neural circuit function prior to trauma exposure predict animals' susceptibility to developing PTSD-like behaviors. This suggests that such pre-existing traits may be critical for determining individual resilience/susceptibility to developing PTSD.

\section{Results}

Paradigm. The experimental schedule is summarized in Fig. 1a. Rats were first scanned using rsfMRI, then exposed to fox urine, and then given an anxiety test approximately one week after exposure.

Freezing responses to fox urine were variable across animals. A total of 87 Long-Evans (LE) rats were exposed to a predator scent stressor in the form of a compressed cotton pad sprayed with fox urine in an inescapable cage for $10 \mathrm{~min}$. Freezing and location within the cage were quantified with behavioral tracking software (ANY-maze, Stoelting Co., VA). Compared to control animals that were exposed to a clean cotton pad, the group exposed to predator scent showed increased freezing ( $t$-test, $t_{87}=3.660, p=$ 0.00044 , Fig. 1b, left), and maintained a significantly greater distance from the cotton pad ( $t$-test, $t_{87}=3.568, p=0.00060$, Fig. $1 \mathrm{~b}$, right, cage length $=45 \mathrm{~cm})$. These data confirm that predator scent exposed animals displayed a significantly greater stress response compared to controls.

Closer examination of animals' freezing time revealed distinct distributions between groups. Figure 1c showed the histograms of freezing time in both groups. Control animals exhibited a unimodal distribution of freezing, whereas predator scent exposed animals displayed large inter-subject variability with a bimodal distribution (Fig. 1c, right panel) fitted with a mixed Gaussian distribution (bimodal Gaussian function provided the best fit relative to uni-modal and tri-modal Gaussian functions, model fit quality (the model with the lowest AIC is the best model to fit the data): $\Delta_{1}(\mathrm{AIC})=11, \Delta_{2}(\mathrm{AIC})=0, \Delta_{3}(\mathrm{AIC})=6$; Akaike weight for each model: $\left.w_{1}=0.0039, w_{2}=0.947, w_{3}=0.0488\right)$. To further investigate this variation in freezing behavior, predator scent exposed animals in the top and bottom tertiles of freezing time were separated into high-freezing and low-freezing subgroups, respectively ( $n=21$ for each subgroup). Figure $1 \mathrm{~d}$ plotted freezing time as a function of exposure time in high-freezing, low-freezing and control groups. Interestingly, freezing differed between high- and low-freezing animals even during the first minute of predator scent exposure (two-sample $t$-test, $p<0.009$ ) and this difference became larger over the time course of exposure. There was no difference between low-freezing rats and controls. There was also no significant difference in mobility among all three groups, as evidenced by the maximum speed during exposure (one-way ANOVA, $F_{(2,62)}=0.86, p=0.43$, Supplementary Fig. 1).

Low, but not high freezers developed PTSD-like behaviors. The fact that freezing time was almost identical between low-freezing and control animals might suggest that low-freezing animals had a similar stress response to controls. However, the spatial distribution of time spent in the cage during exposure suggested a different interpretation (Fig. 2). Control animals did not show a side preference with a near normal distribution of the time spent close to the center of the cage (Fig. 2a). In contrast, both highand low-freezing animals exhibited a leftward skewed distribution of time spent, farther away from the fox urine. The average distance from the cotton pad was significantly greater in both lowand high-freezing animals compared to controls (One-way ANOVA, $F_{(2,62)}=10.97, p=0.000050$ and $p=0.031$, respectively), indicating avoidance behavior in exposed animals. This difference was also supported by the normalized freezing time at different distances from the cotton pad, as shown in Fig. $2 b$. Similar to the distribution of the total time spent in the cage, control animals did not seem to have any preference for the side of the cage in which they froze, whereas both high- and lowfreezing animals tended to freeze farther away from the fox urine 
Experimental schedule diagram
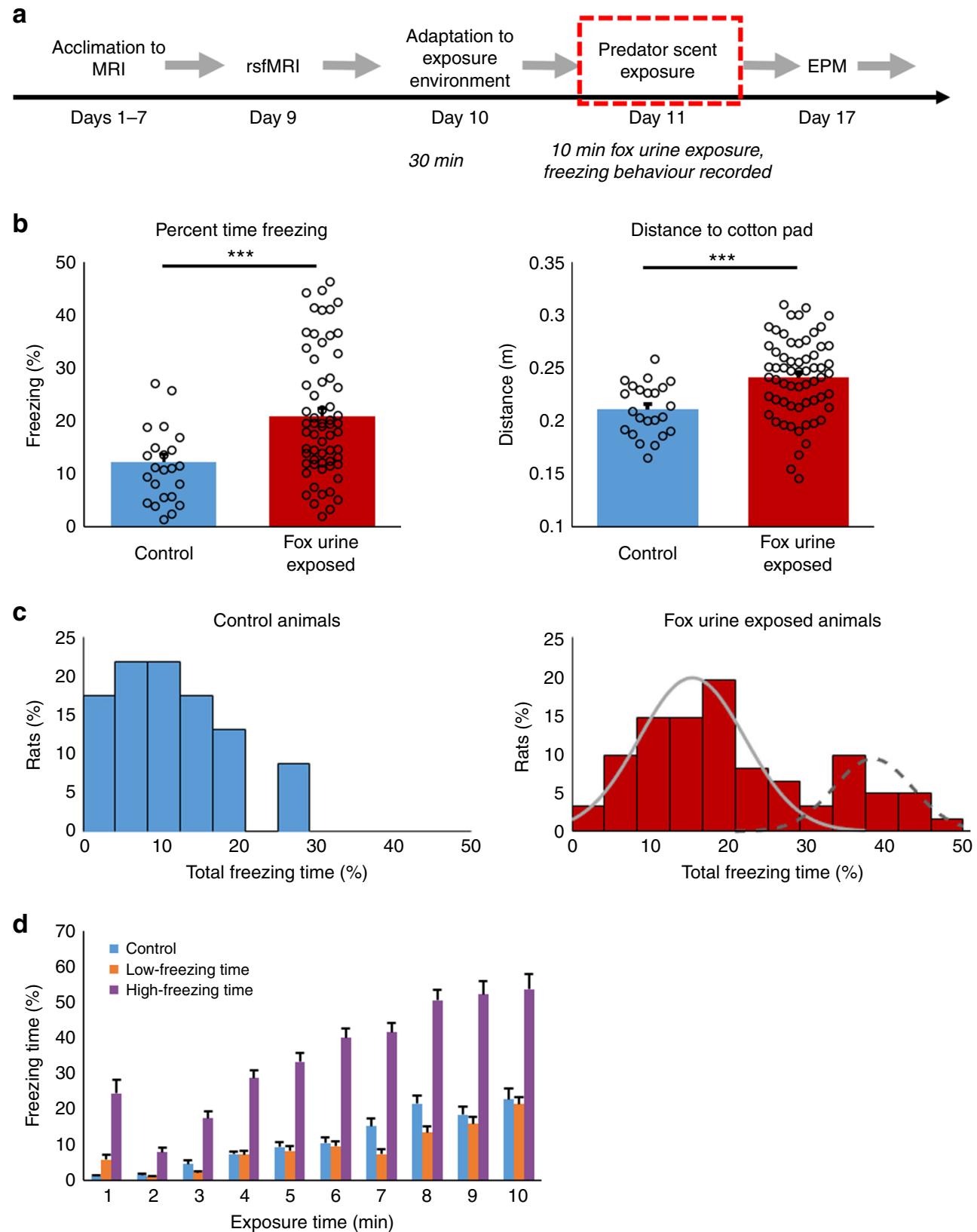

Fig. 1 Freezing behavior during exposure. Freezing time during the 10-min exposure session in control $(n=23)$ and fox-urine exposed $(n=62)$ groups. a Diagram of experimental schedule. b Fox-urine exposed animals showed a significantly longer freezing time (left, $p<0.0005$, in percentage) and greater mean distance (right, $p<0.0007$, in meters) from the cotton pad than controls. c The distributions of individual animals' freezing time in the control group (left) and the exposed group (right) displayed as probability histograms. The freezing time distribution in exposed animals was fit to a Gaussian mixture model, which demonstrated a bimodal distribution. d Freezing time each min plotted as a function of time in low-freezing $(n=21)$, high-freezing $(n=21)$ and control animals during fox-urine exposure. The high-freezing animals displayed higher freezing phenotype during the first minute of exposure, with a significantly longer freezing time than both low-freezing and control animals from the 1st minute through the 10th minute ( $p<0.005$ for each minute of exposure). Bars indicate s.e.m. Source data of Fig. 1b-d are provided as a Source Data file

sample. Relative to high-freezing animals, low-freezing animals showed even more leftward bias. The normalized freezing time was significantly less in the zone $24 \mathrm{~cm}$ away from the pad (Twoway Repeated ANOVA, $F_{\text {Group } \times \text { Zone }}(22,682)=3.00, p=$ 0.000006; Tukey-Kramer post-hoc test, low- vs. high-freezing at $24 \mathrm{~cm}: p=0.04$ ), indicating that low-freezing animals were somewhat more avoidant than high-freezing animals. This can also be seen in the heat plots of Fig. 2a.

Prior to predator scent exposure during the habituation period, the three groups showed no significant differences in freezing time (One-way ANOVA, $F_{\text {Group } \times \text { Zone }}(2,62)=0.24, p=0.788$, Supplementary Fig. 2) or location distribution (the cage was divided into three even-size zones, Two-way ANOVA, $\left.F_{\text {Group } \times Z \text { one }}(4,186)=1.28, p=0.28\right)$. In addition, the three groups exhibited no differences in total distance traveled (oneway ANOVA, $F(2,62)=0.082, p=0.92$ ), maximum speed (oneway ANOVA, $F(2,62)=0.0085, p=0.99)$, or average speed while mobile (one-way ANOVA, $F(2,62)=0.21, p=0.81$ ) during the pre-exposure period, further confirming consistent mobility among these animals. 
a

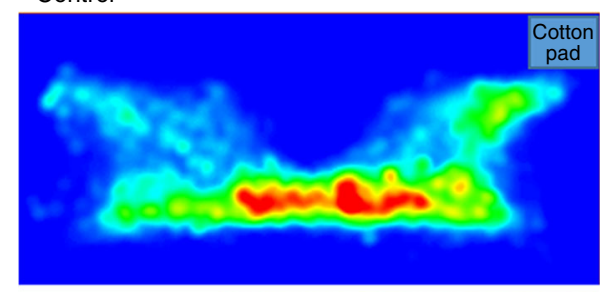

Time spent: $0 \mathrm{~s} \quad \sim 5.0 \mathrm{~s}$

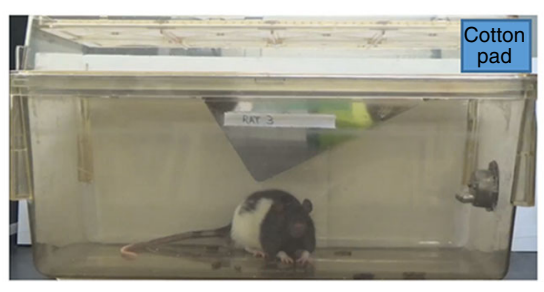

b

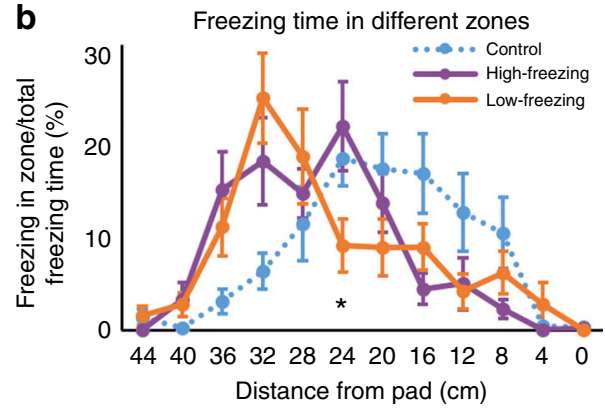

d

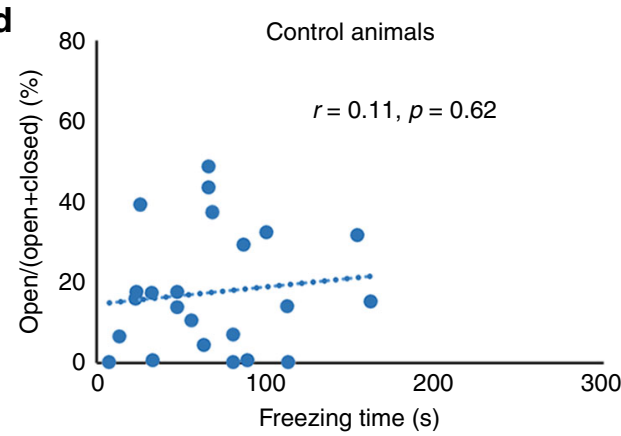

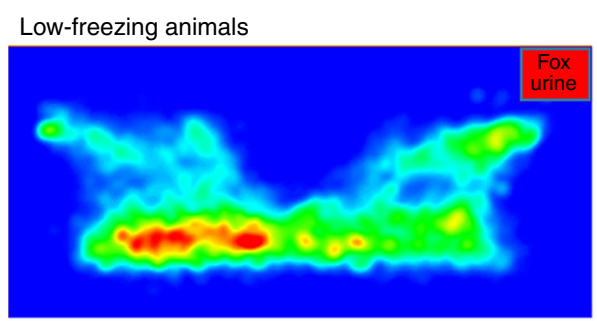

High-freezing animals
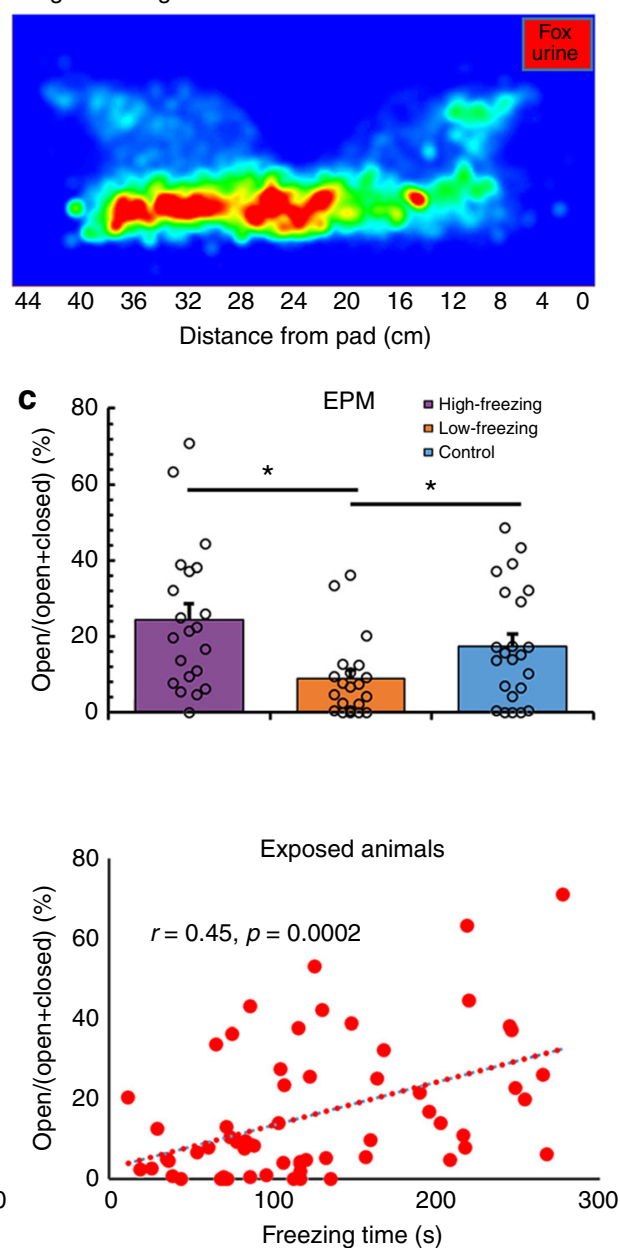

Fig. 2 Stronger stress response and higher long-lasing anxiety in low-freezing animals. a Heat maps of the total time at each location for the control, lowfreezing, and high-freezing groups. The control group showed no evident bias for either side of the cage. The low-freezing group showed a strong bias toward the far end (from the pad) of the cage, while the high-freezing rats also showed a bias toward the far end of the cage, but to a lesser extent. $\mathbf{b}$ The distribution of the percent freezing time for each group across the cage, divided into 12 equivalent-sized zones. The $x$-axis indicates the distance to the pad from the center of each zone. Similar to the distributions of total time spent, the percent freezing time in control rats displayed little bias for either side of the cage. High- and low-freezing animals showed a bias of percent freezing time toward the far end of the cage, with the low-freezing rats showing even stronger bias than high-freezing animals, evidenced by significantly lower percent freezing time of the low-freezing group in the zone $24 \mathrm{~cm}$ away from the pad compared to the high-freezing group $(p=0.04)$. c Elevated plus maze performed 6 days post exposure in all three groups of animals. Low-freezing animals showed a significantly lower EPM score (open/open + closed arm time) compared to both control ( $p=0.030$ ) and high-freezing animals ( $p=$ 0.0017), while no significant difference was seen between control and high-freezing groups. $\mathbf{d}$ The freezing time was positively correlated to the EPM score across all exposed animals ( $r=0.45, p=0.0002)$, but not across control animals $(r=0.11, p=0.62)$. Bars indicate s.e.m. Source data of Fig. 2b-d are provided as a Source Data file

To assess the long-lasting PTSD-like behaviors in exposed animals, we measured anxiety levels using an elevated plus maze (EPM) 6 days after predator scent exposure. Resembling the observation of increased avoidance, low-freezing animals exhibited heightened long-lasting anxiety compared to both control ( $t$ test, $t=2.24, p=0.030)$ and high-freezing animals ( $t$-test, $t=$ $3.37, p=0.0017)$, as indicated by the ratio of open/open + close arm time in EPM tests (Fig. 2c). There was no statistical difference between high-freezing and control animals, indicating that low-, but not high-freezing animals were susceptible to developing PTSD-like behaviors following exposure to predator scent. In addition, freezing was positively correlated with the EPM score in exposed animals $\left(R^{2}=0.21, p=0.0002\right)$, but not controls $\left(R^{2}=0.01, p=0.62\right.$, Fig. $\left.2 \mathrm{~d}\right)$. These results demonstrate 
a
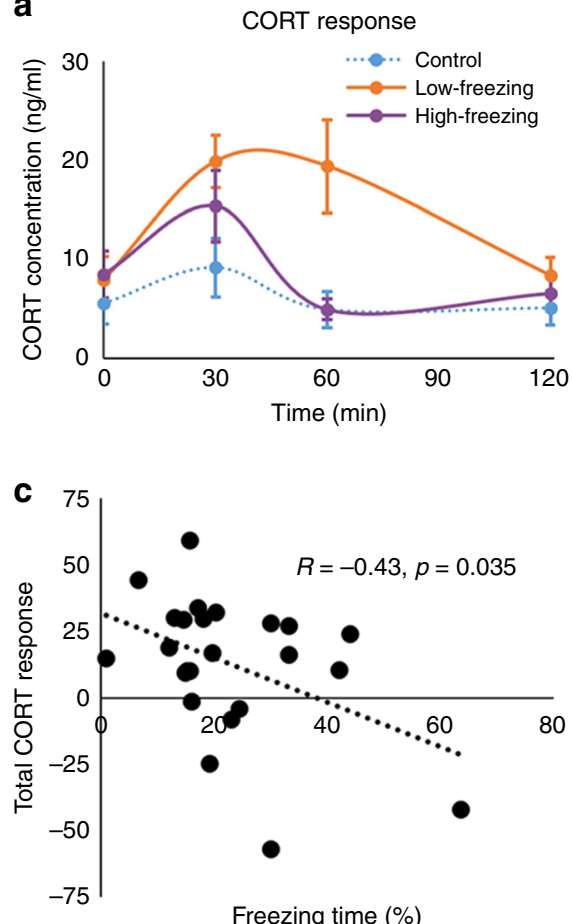

b
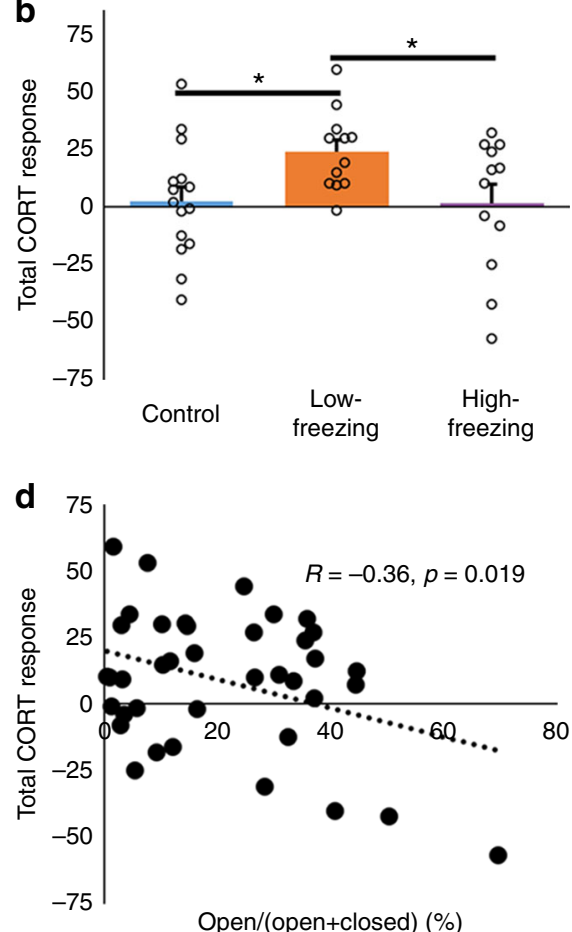

Fig. 3 Corticosterone (CORT) responses. a Time course of CORT (in $\mathrm{ng} / \mathrm{ml}$ ) for high-, low-freezing, and control animals. There was a significant difference in CORT level changes over time among three groups (Two-way repeated ANOVA, $F_{\text {group } \times \text { time }}(6,108)=2.84, p=0.023$ ). Control animals showed no appreciable CORT change across time (One-way repeated ANOVA, $F_{\text {time }}(3,56)=0.8, p=0.50$ ). High-freezing animals exhibited a relatively short CORT response, peaking at $30 \mathrm{~min}$ and returning to baseline at $60 \mathrm{~min}$ (Tukey-Kramer test, $30 \mathrm{~min}$ vs. $60 \mathrm{~min}: p=0.049 ; 0 \mathrm{~min}$ vs. $60 \mathrm{~min}: p=0.54 ; 60 \mathrm{~min}$ vs. 120 min: $p=0.93$ ). The peak amplitude of CORT response at 30 min was comparable between high- and low-freezing animals (Tukey-Kramer test, $p=$ 0.59). However, relative to high-freezing rats, the CORT response in low-freezing animals was prolonged, maintained at a high level at 60 min (TukeyKramer test, $30 \mathrm{~min}$ vs. $60 \mathrm{~min}: p=1 ; 0 \mathrm{~min}$ vs. $60 \mathrm{~min}: p=0.0005$ ) and had a delayed return to baseline at 120 min (Tukey-Kramer test, 60 min vs. 120 min: $p=0.0098 ; 0$ min vs. 120 min: $p=1$ ). In addition, low freezers' CORT level was significantly higher at 60 min than high freezers (Tukey-Kramer test, $p=0.0039)$. $\mathbf{b}$ Low-freezing animals showed a significant higher total CORT response than high-freezing $(p=0.031)$ and control $(p=0.017)$ animals. c Significant correlation between the total CORT responsive ( $y$-axis) and freezing time ( $x$-axis) during exposure across all fox-urine exposed animals $(r=$ $-0.43, p=0.035)$. d Significant correlation $(r=-0.36, p=0.019)$ between the total CORT response ( $y$-axis) and EPM score (open/open + closed arm time, $x$-axis) measured 6 days post exposure across all animals tested. Bars indicate s.e.m. Source data of Fig. 3 are provided as a Source Data file

that behavioral responses during trauma exposure can predict long-term maladaptation. Furthermore, in a separate experiment (Supplementary Fig. 3) low-freezing animals were also associated with other types of PTSD-like behaviors, including increased marble burying behavior ( $n=5$ each group, unpaired two-sample $t$-test, $t=5.47, p=0.048)$, as well as altered habituation to acoustic startle response $(n=5$ each group, two-sample $t$-test, $t$ $=5.3, p=0.050)$ and pre-pulse inhibition $(n=9$ each group, two-sample $t$-test, $t=5.16, p=0.037$ ) relative to high-freezing animals 6 days post predator scent exposure. These data provide additional evidence that low freezers were vulnerable to developing PTSD-like behaviors.

Low-freezing rats showed prolonged corticosterone response. We also measured the corticosterone (CORT) level immediately before predator scent exposure ( $0 \mathrm{~min}$, baseline), as well as 30,60 , and $120 \mathrm{~min}$ after exposure (Fig. 3, 15 controls, including nine exposed to air and six exposed to a neutral scent (lemon), and 24 fox-urine exposed animals). Again, all predator scent exposed animals $(n=24)$ were evenly divided to 12 low-freezing and 12 high-freezing animals according to their freezing time during exposure (we did not use tertiles given a relatively smaller sample size in this experiment).

There was a significant difference in CORT level changes over time among groups (Two-way repeated ANOVA, $F_{\text {group } \times \text { time }}$ $(6,108)=2.84, p=0.023$, Fig. 3a). In particular, control animals showed no significant CORT change across time (One-way repeated ANOVA, $\left.F_{\text {time }}(3,56)=0.8, p=0.50\right)$. High-freezing animals exhibited a relatively short CORT response, peaking at $30 \mathrm{~min}$ and returning to baseline at $60 \mathrm{~min}$ (Tukey-Kramer test, $30 \mathrm{~min}$ vs. $60 \mathrm{~min}: p=0.049$; $0 \mathrm{~min}$ vs. $60 \mathrm{~min}: p=0.54 ; 60 \mathrm{~min}$ vs. 120 min: $p=0.93$ ). The peak amplitude of CORT response at 30 min was comparable between high- and low-freezing animals (Tukey-Kramer test, $p=0.59$ ). However, relative to high-freezing rats, the CORT response in low-freezing animals was prolonged, maintained at a high level at $60 \mathrm{~min}$ (Tukey-Kramer test, $30 \mathrm{~min}$ vs. 60 min: $p=1 ; 0$ min vs. 60 min: $p=0.0005)$ and showed a delayed return to baseline at $120 \mathrm{~min}$ (Tukey-Kramer test, $60 \mathrm{~min}$ vs. $120 \mathrm{~min}: p=0.0098$; $0 \mathrm{~min}$ vs. $120 \mathrm{~min}: p=1)$. In addition, low freezers' CORT level was significantly higher at 60 min than high freezers' (Tukey-Kramer test, $p=0.0039$ ).

The total CORT response was quantified by summing the CORT amplitude from all time points with the baseline CORT level (i.e., $0 \mathrm{~min}$ ) being subtracted from each time point. This quantification method took into account both the amplitude and duration of the hormone response. Low freezers showed significantly higher total CORT response than high freezers $(t=5.29, p=0.031$, Fig. $3 \mathrm{~b})$, as well as controls $(t=6.58, p=$ 0.017, Fig. 3b). Furthermore, the total CORT response was significantly correlated with freezing time across all fox-urine exposed animals $(R=-0.433, p=0.035, n=24$, Fig. $3 c)$, and was also linked to long-lasting anxiety, evidenced by a significant 
correlation with the EPM score measured 6 days after exposure across all animals tested ( $r=-0.36, p=0.019, n=39$, Fig. $3 \mathrm{~d})$. Taken together, these data demonstrate that low-freezing animals displayed prolonged CORT response to predator scent stress, which was related to long-term anxiety.

Pre-trauma neural circuit function predicted fear responses. rsfMRI was performed before trauma exposure to measure preexisting resting-state functional connectivity (RSFC) in neural circuits across the whole brain. Echoing our behavioral data showing difference in freezing during the very beginning of predator scent exposure, RSFC of multiple widespread neural circuits significantly differed between high- and low-freezing animals (false discovery rate $(F D R)=0.01$, Supplementary Fig. 4a). These regions/circuits have been implicated in stress responses, processing of emotionally valent stimuli, olfactory function and freezing behavior.

To identify the specific neural circuits correlated with the freezing phenotype, we conducted a data-driven correlational analysis for all neural connections (67 regions covering the whole brain, 2211 connections in total) between their RSFC and the cumulative freezing time across all exposed animals (Fig. 4a, upper triangle). 15 neural circuits exhibited a significant correlation between pre-exposure RSFC and freezing time $(p<$ 0.05 , FDR corrected, Fig. 4a, lower triangle). Relative to highfreezing animals, six of these connections showed stronger connectivity, whereas nine showed lower connectivity in lowfreezing animals, suggesting that the pre-trauma connectivity within these circuits might have predictive value for the freezing behavior during exposure. Figure $4 \mathrm{~b}$ showed three representative RSFC maps, respectively generated with one region in these 15 connections as the seed (i.e., anterior cingulate cortex (ACC), anterior olfactory nucleus (AON) and parabrachial nucleus $(\mathrm{PBn})$ ). Figure 5 showed the correlational relationship between pre-trauma RSFC and freezing time for all 15 circuits. The diagram of this freezing-associated network was summarized in Fig. 6.

A closer examination of these 15 circuits revealed a comprehensive network particularly involving the olfactory and stress-related brain areas. Specifically, low-freezing animals showed stronger pre-trauma connectivity in two olfactory circuits (i.e., AON-amygdala, and olfactory bulb-auditory cortex), two septal circuits (i.e., lateral septum (LS)-bed nucleus of the stria terminalis (BNST), medial septum (MS)-prelimbic cortex (PL)), and two circuits within the stress system (lateral hypothalamuscortical amygdala and lateral hypothalamus-amygdala). Stronger connectivity in the two olfactory circuits suggest that odor may exert more influence on the amygdala and cortex in low-freezing animals. Additionally, the LS-BNST projection might underlie stronger predator scent-induced stress response in low-freezing animals. In support of this, both the LS and BNST are activated by predator odor ${ }^{24,25}$, and the BNST is actively involved in sustained fear response to diffuse threat ${ }^{26}$. Stronger functional connectivity within the stress system can also contribute to higher stress response and anxiety in low-freezing animals.

Conversely, nine neural circuits showed weaker pre-trauma functional connectivity in low-freezing animals, including two parabrachial nucleus (PBn) circuits (PBn-BNST and PBn-medial hypothalamus), two nucleus accumbens (NAc) circuits (NAcamygdala and NAc-visual cortex), two zona inserta (ZI) circuits (ZI-pituitary gland and ZI-visceral cortex), as well as the circuits of lateral olfactory tract nucleus (nLOT)-anterior cingulate cortex (ACC), postsubiculum-agranular insula (AI), and globus pallidus (GP)-paraventricular hypothalamus (PVN). PBn is tightly related to olfactory function and electrophysiological data show that calcitonin gene-related peptide (CGRP) in PBn has inhibitory effects in the $\mathrm{BNST}^{27}$. Therefore, weaker connectivity in the PBnBNST circuit suggests less inhibition in BNST and could contribute to heightened predator scent-induced anxiety in lowfreezing animals. Weaker RSFC between the nLOT and ACC can reduce ACC's control over predator scent-triggered defense reaction in low-freezing animals. In addition, the NAcamygdala circuit mediates avoidance behaviors ${ }^{28}$, and decreased NAc connectivity was consistent with the report of the inverse association between NAc activity and emotional stress response ${ }^{24}$, which might also be related to goal-directed motor behavior ${ }^{29}$. Furthermore, lower connectivity in ZI circuits (i.e., ZI-pituitary gland and ZI-visceral cortex) may be involved in control of sympathetic visceral reactions and the hypothalamic-pituitaryadrenal (HPA) axis activity ${ }^{30}$. Lastly, the GP-PVN hypothalamus projection, known to involve corticotropin-releasing factor (CRF) neurons, was reported to link stress response to motor output ${ }^{31}$.

Taken together, these data indicate that differences in functional connectivity in neural circuits particularly involving the olfactory and stress-related systems might predispose animals to differential stress responses during predator scent exposure, which can be linked to individual difference in subsequent development of PTSD-like behaviors long after trauma exposure.

Fear responses were not due to novelty of the scent. To rule out the possibility that differential fear responses in fox-urine exposed animals resulted from the novelty of the scent, we repeated the experiment with a lemon scent as the control scent in a separate cohort of animals $(n=24)$. Lemon exposed animals showed virtually identical freezing (Supplementary Fig. 5a, left, $n=6$, two-sample $t$-test, $t=2.0, p=0.16$ ) and avoidance (distance from the pad, Supplementary Fig. 5a, right, two-sample $t$-test, $t=1.14$, $p=0.32$ ) compared to animals exposed to no scent (i.e., air, $n=$ 6 ), which ruled out the possibility that the novelty nature in the scent affected fear responses during exposure. In contrast, the fox-urine exposed group $(n=12)$ maintained a greater mean distance from the cotton pad (one-way ANOVA, $F_{(2,23)}=11.61$, $p=0.0004)$ than both air $(p=0.012)$ and lemon $(p=0.0006)$ exposed animals. In addition, fox-urine exposed animals showed a trend of more freezing than non-traumatized animals (left, $p=$ 0.056 , lemon and air groups combined). Furthermore, consistent with the findings in the main cohort of animals, low-freezing animals exhibited stronger stress response during fox-urine exposure and higher anxiety 6 days after exposure (Supplementary Fig. 5b-d). Taken together, these results ruled out the possibility that differential short-term and long-term behavioral responses in fox-urine exposed animals was due to the novel nature of the fox-urine scent.

Differential fear behaviors were not caused by imaging. To rule out the possibility that differential fear response during predator scent exposure and long-term anxiety between low- and highfreezing animals indeed resulted from predator scent exposure as opposed to other factors, such as the acclimation and/or imaging procedures, we also divided control animals into tertiles based on their cumulative freezing time. No differences in avoidance behaviors, including distance to the cotton pad (two-sample $t$ test, $t=1.22, p=0.25$ ), the distribution of time spent in the cage (two-way ANOVA, $F_{2,28}=2.20, p=0.13$, Supplementary Fig. 6a), the distribution of freezing time in the cage (repeated measures ANOVA, $F_{2,154}=0.34, p=0.98$ ), or long-term anxiety level (EPM score, two-sample $t$-test, $t=0.37, p=0.72$ ) were observed between low- and high-freezing control animals. In addition, unlike predator scent exposed animals, no neural connections exhibited correlation between pre-exposure RSFC and freezing 
a

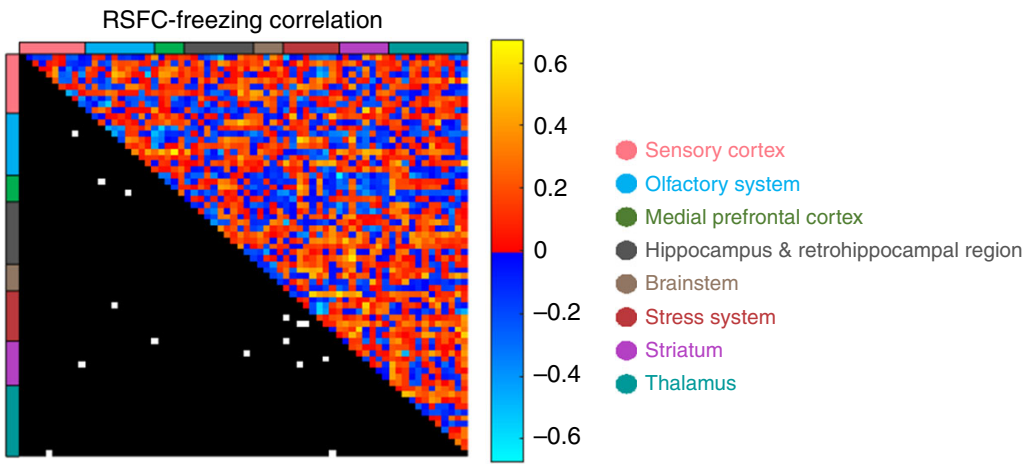

b

Low-freezing group
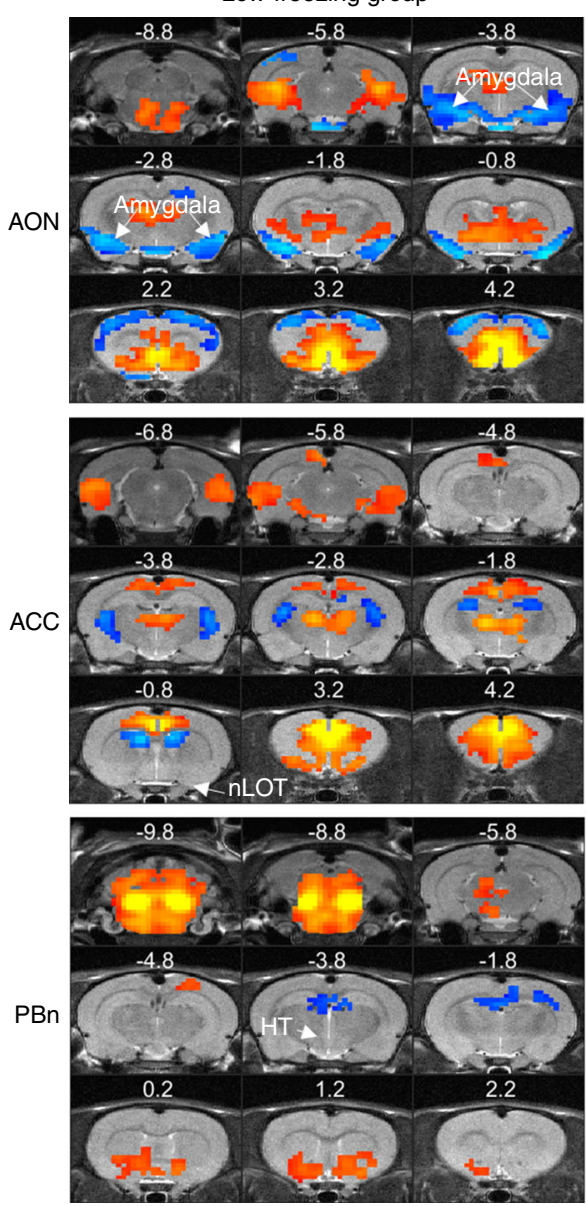

High-freezing group
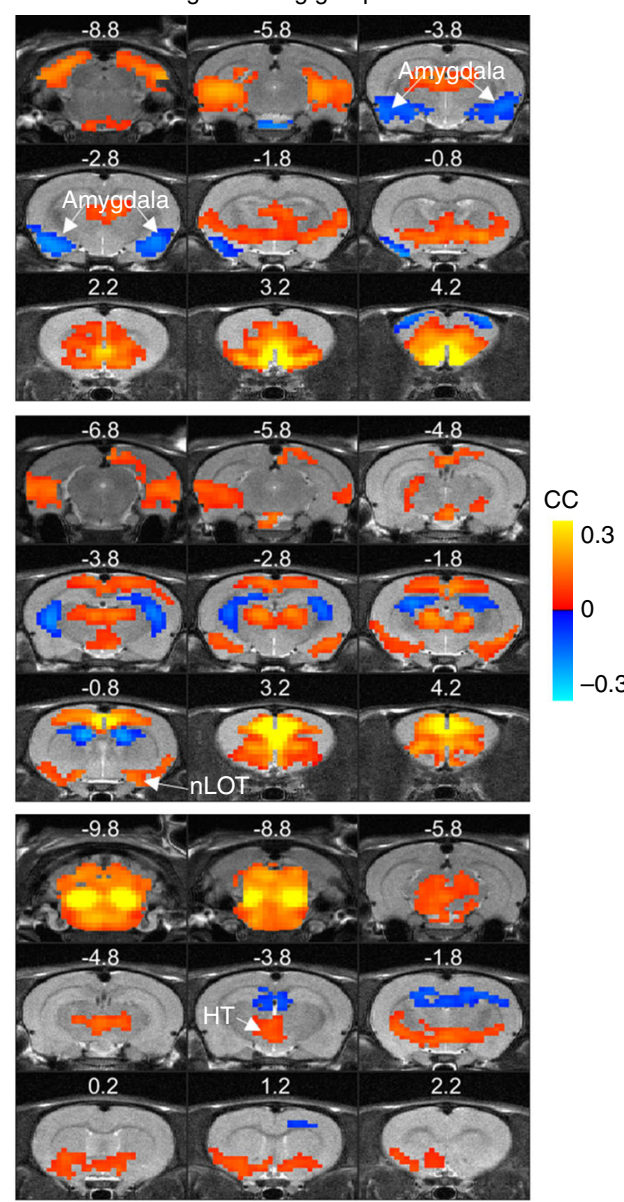

Fig. 4 Pre-trauma functional connectivity correlated to differential fear responses. a Correlations between pre-trauma RSFC and freezing time for all connections (upper triangle) and super-threshold connections (lower triangle, each white element represents a supra-threshold connection). Brain regions are arranged based on the brain system (color coded) they belong to. $\mathbf{b}$ Three representative RSFC maps, generated with ACC, AON, and PBn as the seed, respectively (colored voxels, $p<0.05$, FDR corrected). Distance to Bregma was labeled at the top of each slice. ACC, anterior cingulate cortex; AON, anterior olfactory nucleus; HT, hypothalamus; nLOT, lateral olfactory tract nucleus; PBn, parabrachial nucleus

time, and no connection displayed a significant RSFC difference between low- and high-freezing control animals (Supplementary Fig. $4 \mathrm{~b}$ ), indicating that these animals showed virtually no difference in neural circuit function.

Furthermore, we repeated the fox-urine exposure experiment in a separate cohort of animals that were not previously acclimated or imaged (control, $n=20$, fox-urine exposed, $n=$ 35). Similar differential response during exposure was found in these animals, with low freezers generally exhibiting more avoidance from the fox urine (Supplementary Fig. 6b). These data suggest that the inherent difference between low and high freezers was not caused by potential differential responsiveness to acclimation. This result was supported by the data showing that all three groups of animals (i.e., low-freezing, high-freezing, and control) in the main cohort were acclimated to a similar degree, reflected by their consistent motion levels during imaging (Oneway ANOVA, $F_{(2,62)}=1.23, p=0.3$, Supplementary Fig. $4 c$ ). These findings are also consistent with our previous publications demonstrating that acclimation/imaging does not mask, nor does it interact with the effect of predator stressor $21,23,32$. Taken together, these results confirmed that differential fear behaviors and anxiety levels between low- and high-freezing animals were 
Pre-existing neural circuits associated with freezing

Stronger connectivity in low-freezing animals
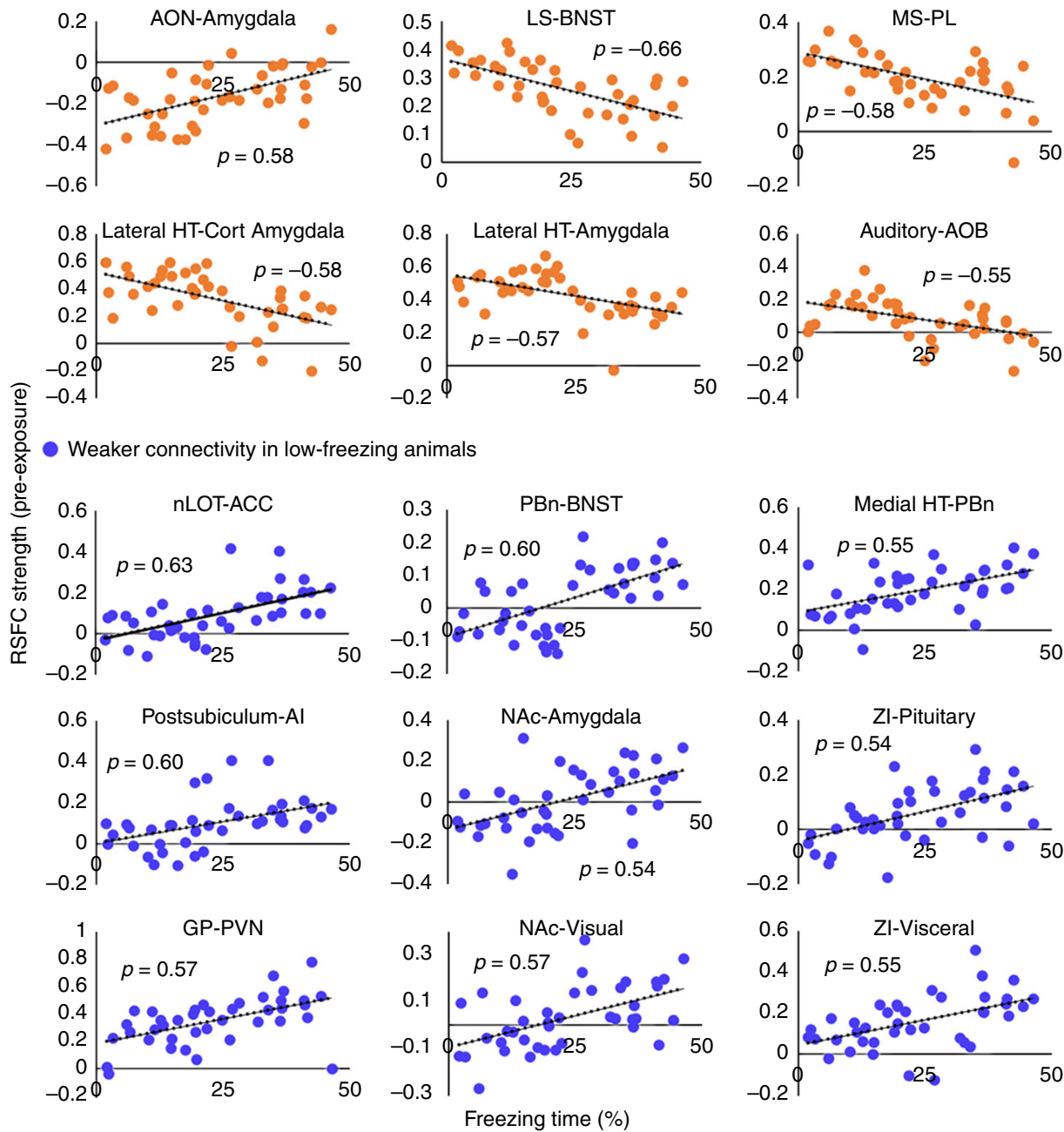

Fig. 5 Correlations between pre-existing functional connectivity and freezing. Neural circuits with pre-trauma RSFC ( $y$-axis) correlated ( $p<0.05$, FDR corrected) with freezing time ( $x$-axis) in all fox-urine exposed animals. AON, anterior olfactory nucleus; LS, lateral septum; BNST, bed nucleus of the stria terminalis; MS, medial septum; PL, prelimbic cortex; HT, hypothalamus; Cort Amygdala, cortical amygdala; AOB, accessory olfactory Bulb; nLOT, lateral olfactory tract nucleus; ACC, anterior cingulate cortex; PBn, parabrachial nucleus; Al, agranular insula; NAc, nucleus accumbens; ZI, zona inserta; GP, globus pallidus; PVN, paraventricular nucleus. Source data of Fig. 5 are provided as a Source Data file

not driven by the acclimation/imaging procedures as all control animals underwent the identical procedures, including imaging, except for being exposed to predator scent.

\section{Discussion}

Employing a longitudinal design, we investigated animals' individual variability in stress response and subsequent development of PTSD-like behaviors following predator scent exposure. We found that animals displayed large differences in freezing behavior during fox-urine exposure. Animals expressing less freezing exhibited increased avoidance and tended to develop long-lasting heightened anxiety. Importantly, this difference in behavioral responses was apparent at the beginning of predator scent exposure, suggesting pre-existing vulnerability. This notion was supported by the rsfMRI data showing significant pre-trauma difference in neural circuit connectivity between high- and lowfreezing animals. In addition, low-freezing animals showed a prolonged CORT response to predator scent, which was correlated with freezing and long-lasting EPM anxiety measurement. Taken together, this study provides important evidence supporting the hypothesis that pre-existing circuit function determines the defense response strategy during threat and that this behavioral response might be directly related to the development of PTSD. It also highlights the importance of analyzing factors both before and after trauma for the study of PTSD.

Behavioral responses to stress are commonly categorized into fight, flight or freezing. In rodent behavior testing, freezing is dominantly used as the measure of threat reaction. Although freezing is a universal fear response, it manifests at intermediate levels of predator threat ${ }^{16}$. Meanwhile, other types of threatinduced responses, including fight-or-flight, which is elicited during imminent threat ${ }^{15}$, and avoidance, can counteract the measure of freezing. For this reason, the use of freezing as the only measure for stress response of an individual animal can be problematic if alternative coping strategies are not considered ${ }^{33}$. Indeed, there is growing evidence, suggesting the ambiguity of freezing time as it relates to stress ${ }^{15,34}$.

In line with this concept, our data show that animals with the lowest freezing time are in fact the most stressed subgroup. Their highly biased distribution in the total time and freezing time at 

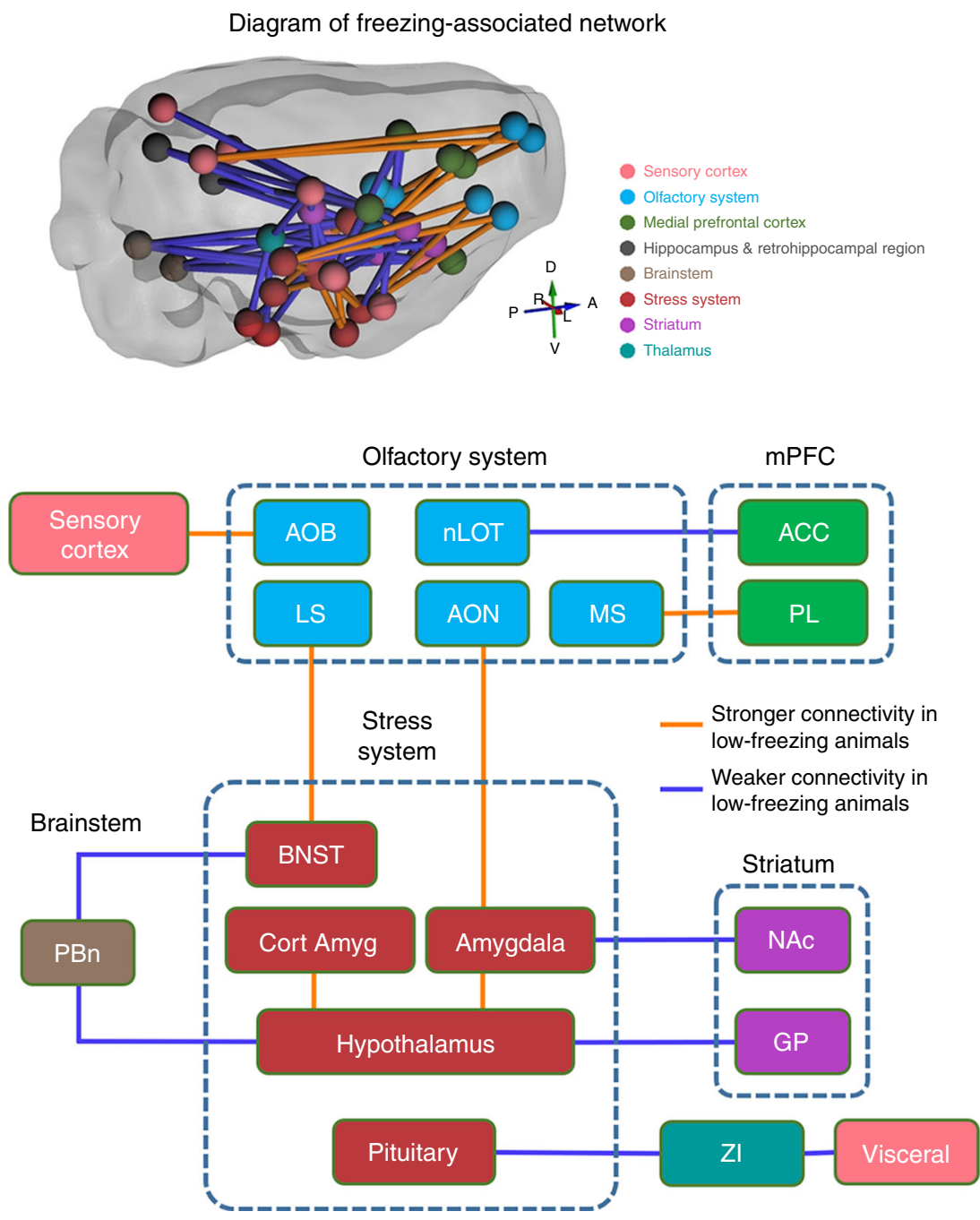

Fig. 6 Diagram of freezing-associated neural network. The diagram includes neural circuits showing significant correlation between pre-trauma RSFC and total freezing time shown in Fig. 5. Brain regions are grouped and color coded based on the brain systems they belong to. Orange lines indicate stronger connectivity in low-freezing animals. Blue lines indicate weaker connectivity in low-freezing animals. See the caption of Fig. 5 for the abbreviation

different distance to the cotton pad indicate that low-freezing animals might have perceived a higher threat level and used strategies other than freezing (e.g., avoidance), particularly at closer proximity to the pad. This result was corroborated by significantly higher total CORT response during exposure in lowfreezing animals, relative to both high-freezing and control animals. This higher perceived threat level is likely a crucial factor in maladaptation to stress response (e.g., higher long-lasting anxiety level), which is in line with the literature investigating the role of threat perception in human PTSD diagnoses ${ }^{35}$. Taken together, these results demonstrate that freezing time alone might not be a reliable measure of reaction to threat. These data also highlight the importance of separating subpopulations, in particular in stress studies, as key information can be lost through the averaging of subpopulations that display bidirectional responses.

It has to be noted that lower freezing behavior in low freezers did not reflect a reduced ability to respond to the predator scent. Low-freezing animals exhibited more locomotor activity, measured by distance traveled during exposure (Supplementary Fig. 1, One-way ANOVA, $F_{(2,62)}=8.43, p=0.001$, high-freezers vs. low freezers, $p=0.001$ ), and more active avoidance from the fox urine. Furthermore, during exposure there was no difference in mobility among low-, high-freezing and control groups, reflected by the maximal speed (Supplementary Fig. 1), also suggesting that low freezers were not suppressed in their response to the foxurine scent. Different responses during exposure were also not originated from any systematic bias in these measures among animals, because during the habituation period prior to exposure, the three groups showed no difference in freezing time (Supplementary Fig. 2) or location distribution. Thus, the difference in freezing to the predator scent likely reflects a difference in the defensive strategy selected.

Whether defensive reactions predict long-term emotion regulation and/or symptom development has been an unresolved issue, largely due to lack of longitudinal studies that follow-up the relationship between behavioral reactions during trauma exposure and the development of psychopathology ${ }^{15}$. Our longitudinal study demonstrates that freezing during trauma exposure predicts long-term maladaptation in animals, supporting the idea that specific behavioral responses to a threat might predict susceptibility to long-term PTSD-like behaviors.

The relationship between the behavioral reaction during stress and vulnerability to stress maladaptation is corroborated by neuroendocrine measures during and following predator scent stress. We observed that in low-freezing (i.e., vulnerable) animals, the CORT response was prolonged and had a delayed return back to baseline after stress, and this pattern of CORT response has been suggested as a marker of maladaptation to stress in PTSD ${ }^{36}$. 
Correspondingly, in our study, both freezing time and the total CORT response were correlated with the long-term anxiety measure. These data are also consistent with the theory that stress hormone production is an important and protective part of acute stress response triggering negative feedback on CORT production $^{37-40}$. In fact, it has been shown that supplementing CORT at the time of trauma ${ }^{41}$, or immediately after trauma ${ }^{42}$ has protective effects against PTSD symptom development ${ }^{37}$. Taken together, our data indicate that individual variation in behavioral and hormonal responses to stress can be key predictors of the development of emotion regulation and chronic pathological states.

Our rsfMRI data suggest that sensory inputs play an important role in the stress response and subsequent development of PTSDlike behaviors. AON has been shown to be activated by variable stressors, including predator scent ${ }^{43}$. The mitral/tufted (MT) cells in the olfactory bulb send their processes to $\mathrm{AON}^{44}$, which projects to amygdala ${ }^{45}$. This connectivity indicates that the flow of olfactory information through the AON to the amygdala could drive the stress response to predator scent. Stronger AONamygdala connectivity in low-freezing animals suggests that the olfactory cue could have a stronger impact on amygdala activity, which would lead to a stronger stress response. Conversely, weaker nLOT-ACC connectivity in low-freezing animals indicates less ACC control during predator scent exposure, which could affect the switching between freezing and active defense modes ${ }^{46}$. These data also agree with the report that the olfactory cortex and its connections with the stress system are tightly linked to stress hormone responses to predator scent in rodents ${ }^{47}$. Taken together, these results highlight the importance of sensory input in the stress response during predator scent exposure, which also imply that weaker sensory drive in the stress system may help promote resilience to trauma.

In addition to stronger sensory-stress connectivity, stronger functional connectivity within the stress system was observed in low-freezing animals, consistent with their higher stress response and anxiety. In particular, it is known that threat-induced autonomic sympathetic responses are mediated by amygdala projections to the lateral hypothalamus ${ }^{15}$. Stronger connectivity in this circuit could result in higher arousal, higher cardiovascular activity and increased muscle tone during predator odor exposure in low-freezing animals ${ }^{48}$.

Our data also suggest that BNST may be involved in the neural network associated with differential stress response during predator scent exposure. BNST, as a part of the extended amygdala, is well known to be involved in sustained anxiety when a threat is diffuse and/or uncertain ${ }^{26,49}$ such as predator odor ${ }^{25}$. As a result, this region is particularly associated with anxiety ${ }^{50-52}$. The anatomical connections between BNST and LS and PBn have been well documented $27,53,54$, but the specific function of these projections is less well studied. It has been shown that CGRP in the $\mathrm{PBn}$ inhibits neurons of the BNST ${ }^{27}$, and BNST projections to the $\mathrm{PBn}$ dampen anxiety response $\mathrm{e}^{55}$. These data support the idea that a weaker PBn-BNST connection in low-freezing (i.e., vulnerable) animals caused disinhibition of BNST activity, which led to a stronger stress response and heightened long-lasting anxiety. Conversely, LS-mediated activity, possibly again driven by olfactory inputs ${ }^{24}$, can potentiate BNST activity in these animals, and generate stronger stress responses and heightened anxiety.

Another interesting circuit that is coupled to freezing behavior is the PVN-GP connection. CRF neurons in the PVN hypothalamus, amygdala and BNST send synapses to the external GP ${ }^{31}$, which expresses high levels of the primary CRF receptor (i.e., CRFR1). These connections makes GP an entry point connecting the stress system with the basal ganglia, which links the stressrelevant information to the directed movement ${ }^{31}$. Consistent with this notion, our data suggest that the pre-trauma connectivity in this circuit is directly associated with stress-related behaviors in animals.

Stronger connectivity between the MS and PL can be linked to stronger fear in low-freezing animals, given that PL promotes fear expression and avoidance ${ }^{56,57}$. Also considering that septal neurons are known to mediate the generation of theta oscillations $^{58,59}$, the MS-PL connection may contribute to fearassociated theta rhythm in prefronto-amygdalo-hippocampal pathways ${ }^{60}$. In addition, the postsubiculum-insula circuit connectivity could be responsible for differential spatial movement in separate subgroups of animals.

Our neural circuitry and neuroendocrine findings in animals echo PTSD research in humans. Altered sensory function has also been reported in PTSD patients ${ }^{61}$. In addition, a significant number of brain regions identified here (e.g., BNST, PFC) are tightly related to anxiety disorders in humans ${ }^{62}$, although the present study goes beyond static neuroanatomy of individual brain regions and focuses on pre-existing neural circuit function. Furthermore, human data also suggest that a dysregulated CORT response is a predictor of vulnerability to subsequent development of PTSD $39,40,42,63$. Taken together, these results provide compelling evidence supporting the relevance of the present study to human PTSD.

In summary, our data demonstrate that differences in neural circuit function that are present before trauma exposure may predispose animals to specific stress responses and long-term PTSD-like behaviors. Future studies that focus on the causal relationship between the baseline neural circuit function and behavioral responses during trauma exposure and long-term outcome in PTSD-like behaviors will be needed to understand pathological susceptibility to PTSD. In addition, low- and highfreezing animals might differ in other behavioral phenotypes that predict vulnerability in anxiety disorders (e.g., novelty-seeking behaviors $)^{64}$. Future research should also investigate this possibility.

\section{Methods}

Animals. Adult male LE rats (250-350 g) were obtained from Charles River Laboratories (Raleigh, North Carolina site). Rats were housed in pairs in Plexiglas cages with controlled ambient temperature $\left(22-24^{\circ} \mathrm{C}\right)$ on a $12 / 12 \mathrm{~h}$ day-dark schedule. Food and water were provided ad libitum. The main cohort of animals included 87 rats in the present study, in which 23 rats were controls and 64 rats were exposed to predator scent using a single-episode predator scent exposure in an inescapable environment paradigm. Two rats were removed from the exposed group before analysis due to health concerns. All rats were used for behavior tests and rsfMRI experiments. All animal procedures were reviewed and approved by the Institutional Animal Care and Use Committee (IACUC) of the Pennsylvania State University. The experimental procedure was summarized in Fig. 1a.

Single-episode predator scent exposure. Rats were exposed to a single-episode of predator scent stress in an inescapable environment for $10 \mathrm{~min}$. The day prior to exposure, each rat was assigned to its own exposure cage (i.e., a Plexiglas cage with no bedding) and habituated to the cage environment for $30 \mathrm{~min}$. The cage was left soiled after habituation. On the exposure day, the rat was placed in its corresponding cage for a total of $12 \mathrm{~min}$. After $2 \mathrm{~min}$ of adaptation, a cotton pad sprayed with red fox urine was placed at the right end of the cage for another $10 \mathrm{~min}$ (Red fox urine, Wild Life Research Center). The pad was put on a wire mesh inside the cage, right below the lid and out of reach of the rat. The control animal was exposed to the same cotton pad without fox urine. During exposure, the rat was recorded by a video camera. Following predator scent exposure, the rat was returned to his home cage and left undisturbed for 6 days.

Elevated plus maze (EPM). The EPM test was performed to measure the anxiety level 6 days after trauma exposure. The maze consisted of four perpendicular arms forming a cross and elevated $50 \mathrm{~cm}$ from the floor. Two arms were closed, opposed to each other and enclosed by $40-\mathrm{cm}$ high walls. The remaining two arms, also opposed to each other, were completely open. A camera was placed directly above the maze to record the rat movement.

To perform the test, the rat was initially placed in the center of the maze facing one open arm and allowed to freely explore the maze for $5 \mathrm{~min}$. The times spent in 
the open and closed arms were determined. The ratio of time in open arms versus that in both open and closed arms [i.e., open/(open + closed)] was calculated to provide a measure of anxiety level. A lower score of this ratio indicates a higher level of anxiety. The maze was cleaned with a $70 \%$ alcohol solution in water between tests.

CORT Measurement. Rats $(n=39)$ had blood samples collected to quantify the hormonal response to stress. CORT level was measured immediately before predator scent exposure (i.e., $0 \mathrm{~min}$ ) and 30, 60, and $120 \mathrm{~min}$ after exposure to quantify the dynamic response to predator scent stress exposure. Initial blood samples were collected through tail nick, with subsequent time point samples collected by disturbing the tail scab. Samples were collected using heparin coated microtubes with capillary tube inserts (RAM Scientific Safe-T-Fill, Nashville, TN). Samples were centrifuged to obtain plasma and stored at $-20^{\circ} \mathrm{C}$. The plasma CORT level was then quantified using CORT enzyme-linked immunosorbent assay (ELISA) kit (Enzo life sciences Inc. Ann Arbor, MI. Cat ADI-901-097) according to the manufacturer's manual. The total CORT response for each animal was quantified by the summation of the CORT amplitude across all time points with the CORT level at $0 \mathrm{~min}$ (i.e., baseline) being subtracted at each time point. This measurement took into account both the amplitude and duration of the CORT response and represented the total output of the

hypothalamic-pituitary-adrenal axis.

Acclimation and rsfMRI data acquisition. All rats were scanned before the trauma exposure experiment. Before imaging, animals were acclimated to a 'mock" imaging environment for 7 days. This acclimation protocol was employed to diminish the stress and motion of the subject during scans ${ }^{65}$. The rat was first briefly anesthetized with $2-4 \%$ isoflurane in oxygen (3-5 min). The head was restrained with a bite bar, nose bar and side pads that restricted the head motion. A pair of shoulder bars and a body tube maintained the body in place without impeding ventilation. The animal was allowed to regain consciousness by discontinuing isoflurane after being set up in the restrainer, typically in 10 mins. The rat was then placed in a dark ventilated box and the sound of different MRI sequences was played, presenting an environment mimicking MRI scanning. All rats were given 7 daily acclimation sessions with a gradually increasing acclimation period $(15,30,45,60,60,60$, and $60 \mathrm{~min}$ from day 1 to day 7$)$. A similar approach has also been applied by other research groups ${ }^{66-68}$.

At least 2 days after acclimation, MRI scans were performed in a 7-Tesla scanner interfaced with the Bruker console system (Bruker, Germany). The rat was first briefly anesthetized with $2-4 \%$ isoflurane in oxygen and set-up in the same restrainer with a built-in birdcage $1 \mathrm{H}$ radiofrequency volume coil. Anesthesia was then discontinued, and the rat was allowed to regain consciousness and placed into the MRI magnet bore. rsfMRI data were obtained using a single shot gradient-echo echo-planar imaging sequence with the parameters of repetition time $(\mathrm{TR})=1 \mathrm{~s}$, echo time $(\mathrm{TE})=13.8 \mathrm{~ms}, 64 \times 64$ matrix size, 20 slices with $1 \mathrm{~mm}$ thickness, field of view $(\mathrm{FOV})=32 \times 32 \mathrm{~mm}^{2}$, in-plane resolution $=500 \times 500 \mu \mathrm{m}^{2}$. Each rat was scanned for $2400 \mathrm{EPI}$ volumes per scan session. Anatomical images were also acquired using a fast spin-echo sequence with the parameters of TR $=1500 \mathrm{~ms}$; TE $=8 \mathrm{~ms}$; matrix size $=256 \times 256 ; \mathrm{FOV}=3.2 \times 3.2 \mathrm{~cm}^{2}$; slice number $=20$; slice thickness $=1 \mathrm{~mm}$; RARE factor $=8$.

rsfMRI data processing. rsfMRI images were preprocessed by performing alignment using Medical Image Visualization and Analysis (MIVA, http://ccni.wpi.edu/), motion correction using SPM 12 (http://www.fil.ion.ucl.ac.uk/spm/), spatial smoothing (Gaussian kernel: $\mathrm{FWHM}=0.75 \mathrm{~mm}$ ) and temporal smoothing (band-pass filter, cutoff frequencies: $0.01-0.1 \mathrm{~Hz}$ ), and regression of motion parameters and signals from the white matter and ventricles as described in past studies $20,22,23,69$. Before performing these steps, EPI volumes with relative framewise displacement (FD) $>0.2 \mathrm{~mm}$ and their immediate temporal neighbors were first removed ${ }^{70}$. In addition, the first ten volumes of each rsfMRI run were removed to ensure the magnetization was at steady state. rsfMRI runs with $>20 \%$ volumes removed were excluded from further analysis.

Functional connectivity analysis was conducted using region-of-interest (ROI)based and seed-based correlational analysis. The ROI method evaluated RSFC by quantifying the Pearson correlation coefficient of regionally averaged spontaneous BOLD signals between different brain regions. Seed maps were generated by correlating the regionally averaged seed time course with the time course of each brain voxel. A linear mixed effect model was used to determine the overall effect on RSFC of each group of rats, while removing any random effect from different batches of animals.

Reporting summary. Further information on research design is available in the Nature Research Reporting Summary linked to this article.

\section{Data availability}

Data supporting the findings of this manuscript are available from the corresponding author upon reasonable request. Source data underlying Figs. 1b-d, 2b-d, 3 and 5, as well as Supplementary Figs. 1, 2, 3, 4c, 5a, 5c-e, and $6 \mathrm{~b}$ are provided as a Source Data file.
Received: 19 September 2018 Accepted: 8 April 2019

Published online: 30 May 2019

\section{References}

1. Charney, D. S. Psychobiological mechanisms of resilience and vulnerability: implications for successful adaptation to extreme stress. Am. J. Psychiatry 161, 195-216 (2004)

2. Yehuda, R. \& LeDoux, J. Response variation following trauma: a translational neuroscience approach to understanding PTSD. Neuron 56, 19-32 (2007).

3. Feder, A., Nestler, E. J. \& Charney, D. S. Psychobiology and molecular genetics of resilience. Nat. Rev. Neurosci. 10, 446-457 (2009).

4. Gilbertson, M. W. et al. Smaller hippocampal volume predicts pathologic vulnerability to psychological trauma. Nat. Neurosci. 5, 1242-1247 (2002).

5. Bonne, O. et al. Longitudinal MRI study of hippocampal volume in trauma survivors with PTSD. Am. J. Psychiatry 158, 1248-1251 (2001).

6. McEwen, B. S., Nasca, C. \& Gray, J. D. Stress Effects on Neuronal Structure Hippocampus, Amygdala, and Prefrontal Cortex. Neuropsychopharmacology 41, 3-23 (2016).

7. Logue, M. W. et al. Smaller hippocampal volume in posttraumatic stress disorder: A Multisite ENIGMA-PGC Study: subcortical volumetry results from posttraumatic stress disorder consortia. Biol. Psychiatry 83, 244-253 (2018).

8. Yehuda, R. Advances in understanding neuroendocrine alterations in PTSD and their therapeutic implications. Ann. N. Y Acad. Sci. 1071, 137-166 (2006).

9. Milad, M. R. et al. Neurobiological basis of failure to recall extinction memory in posttraumatic stress disorder. Biol. Psychiatry 66, 1075-1082 (2009).

10. Shin, L. M. et al. Exaggerated activation of dorsal anterior cingulate cortex during cognitive interference: a monozygotic twin study of posttraumatic stress disorder. Am. J. Psychiatry 168, 979-985 (2011).

11. Goswami, S., Rodriguez-Sierra, O., Cascardi, M. \& Pare, D. Animal models of post-traumatic stress disorder: face validity. Front. Neurosci. 7, 89 (2013).

12. Deslauriers, J., Toth, M., Der-Avakian, A. \& Risbrough, V. B. Current status of animal models of posttraumatic stress disorder: Behavioral and biological phenotypes, and future challenges in improving translation. Biol. Psychiatry 83, 895-907 (2018).

13. Flandreau, E. I. \& Toth, M. Animal models of PTSD: A critical review. Curr. Topic. Behav. Neurosci., https://doi.org/10.1007/7854_2016_65 (2017).

14. Cohen, H. \& Zohar, J. An animal model of posttraumatic stress disorder: the use of cut-off behavioral criteria. Ann. N. Y Acad. Sci. 1032, 167-178 (2004).

15. Roelofs, K. Freeze for action: neurobiological mechanisms in animal and human freezing. Philos. Trans. R Soc. Lond. B Biol. Sci. 372, https://doi.org/ 10.1098/rstb.2016.0206 (2017).

16. Fanselow, M. S. Neural organization of the defensive behavior system responsible for fear. Psychon. Bull. Rev. 1, 429-438 (1994).

17. Liang, Z., King, J. \& Zhang, N. Uncovering intrinsic connectional architecture of functional networks in awake rat brain. J. Neurosci. 31, 3776-3783 (2011).

18. Liang, Z., King, J. \& Zhang, N. Anticorrelated resting-state functional connectivity in awake rat brain. Neuroimage 59, 1190-1199 (2012).

19. Ma, Z. et al. Functional atlas of the awake rat brain: A neuroimaging study of rat brain specialization and integration. Neuroimage 170, 95-112 (2018).

20. Ma, Z. \& Zhang, N. Temporal transitions of spontaneous brain activity. eLife 7, https://doi.org/10.7554/eLife.33562 (2018).

21. Gao, Y. R. et al. Time to wake up: Studying neurovascular coupling and brainwide circuit function in the un-anesthetized animal. Neuroimage, https://doi. org/10.1016/j.neuroimage.2016.11.069 (2016).

22. Liang, Z., King, J. \& Zhang, N. Intrinsic organization of the anesthetized brain. J. Neurosci. 32, 10183-10191 (2012).

23. Liang, Z., King, J. \& Zhang, N. Neuroplasticity to a single-episode traumatic stress revealed by resting-state fMRI in awake rats. Neuroimage 103, 485-491 (2014).

24. Ubeda-Contreras, J., Marin-Blasco, I., Nadal, R. \& Armario, A. Brain c-fos expression patterns induced by emotional stressors differing in nature and intensity. Brain Struct. Funct. 223, 2213-2227 (2018).

25. Janitzky, K., D’Hanis, W., Krober, A. \& Schwegler, H. TMT predator odor activated neural circuit in C57BL/6J mice indicates TMT-stress as a suitable model for uncontrollable intense stress. Brain Res. 1599, 1-8 (2015).

26. Daldrup, T., Lesting, J., Meuth, P., Seidenbecher, T. \& Pape, H. C. Neuronal correlates of sustained fear in the anterolateral part of the bed nucleus of stria terminalis. Neurobiol. Learn. Mem. 131, 137-146 (2016)

27. Gungor, N. Z. \& Pare, D. CGRP inhibits neurons of the bed nucleus of the stria terminalis: implications for the regulation of fear and anxiety. J. Neurosci. 34, 60-65 (2014).

28. Ramirez, F., Moscarello, J. M., LeDoux, J. E. \& Sears, R. M. Active avoidance requires a serial basal amygdala to nucleus accumbens shell circuit. J. Neurosci. 35, 3470-3477 (2015) 
29. Da Cunha, C., Gomez, A. A. \& Blaha, C. D. The role of the basal ganglia in motivated behavior. Rev. Neurosci. 23, 747-767 (2012).

30. Mitrofanis, J. Some certainty for the "zone of uncertainty"? Exploring the function of the zona incerta. Neuroscience 130, 1-15 (2005).

31. Hunt, A. J. Jr. et al. Paraventricular hypothalamic and amygdalar CRF neurons synapse in the external globus pallidus. Brain Struct. Funct. 223, 2685-2698 (2018).

32. Dopfel, D. \& Zhang, N. Mapping stress networks using functional magnetic resonance imaging in awake animals. Neurobiol. Stress 9, 251-263 (2018).

33. Paré, D. \& Quirk, G. J. When scientific paradigms lead to tunnel vision: lessons from the study of fear. NPJ Sci. Learn. 2, 6 (2017).

34. Gruene, T. M., Flick, K., Stefano, A., Shea, S. D. \& Shansky, R. M. Sexually divergent expression of active and passive conditioned fear responses in rats. eLife 4, https://doi.org/10.7554/eLife.11352 (2015).

35. Holbrook, T. L., Hoyt, D. B., Stein, M. B. \& Sieber, W. J. Perceived threat to life predicts posttraumatic stress disorder after major trauma: risk factors and functional outcome. J. Trauma 51, 287-292, discussion 292-283 (2001).

36. Yehuda, R. Current status of cortisol findings in post-traumatic stress disorder. Psychiatr. Clin. North Am. 25, 341-368, vii (2002).

37. Rao, R. P., Anilkumar, S., McEwen, B. S. \& Chattarji, S. Glucocorticoids protect against the delayed behavioral and cellular effects of acute stress on the amygdala. Biol. Psychiatry 72, 466-475 (2012).

38. McEwen, B. S. et al. Mechanisms of stress in the brain. Nat. Neurosci. 18, 1353-1363 (2015).

39. Yehuda, R., McFarlane, A. C. \& Shalev, A. Y. Predicting the development of posttraumatic stress disorder from the acute response to a traumatic event. Biol. Psychiatry 44, 1305-1313 (1998).

40. Delahanty, D. L., Raimonde, A. J. \& Spoonster, E. Initial posttraumatic urinary cortisol levels predict subsequent PTSD symptoms in motor vehicle accident victims. Biol. Psychiatry 48, 940-947 (2000).

41. Schelling, G. et al. Efficacy of hydrocortisone in preventing posttraumatic stress disorder following critical illness and major surgery. Ann. N. Y Acad. Sci. 1071, 46-53 (2006).

42. Zohar, J. et al. High dose hydrocortisone immediately after trauma may alter the trajectory of PTSD: interplay between clinical and animal studies. Eur. Neuropsychopharmacol.: J. Eur. Coll. Neuropsychopharmacol. 21, 796-809 (2011).

43. Dielenberg, R. A., Hunt, G. E. \& McGregor, I. S. "When a rat smells a cat": the distribution of Fos immunoreactivity in rat brain following exposure to a predatory odor. Neuroscience 104, 1085-1097 (2001).

44. Friedrich, R. W. Olfactory neuroscience: beyond the bulb. Curr. Biol. 21, R438-R440 (2011).

45. Ferrer, N. G. Efferent projections of the anterior olfactory nucleus. J. Comp. Neurol. 137, 309-320 (1969).

46. Gozzi, A. et al. A neural switch for active and passive fear. Neuron 67, 656-666 (2010).

47. Kondoh, K. et al. A specific area of olfactory cortex involved in stress hormone responses to predator odours. Nature 532, 103-106 (2016).

48. Keay, K. A. \& Bandler, R. Parallel circuits mediating distinct emotional coping reactions to different types of stress. Neurosci. Biobehav. Rev. 25, 669-678 (2001).

49. Walker, D. L. \& Davis, M. Role of the extended amygdala in short-duration versus sustained fear: a tribute to Dr. Lennart Heimer. Brain Struct. Funct. 213, 29-42 (2008).

50. Somerville, L. H., Whalen, P. J. \& Kelley, W. M. Human bed nucleus of the stria terminalis indexes hypervigilant threat monitoring. Biol. Psychiatry 68 , 416-424 (2010).

51. Grupe, D. W. \& Nitschke, J. B. Uncertainty and anticipation in anxiety: an integrated neurobiological and psychological perspective. Nat. Rev. Neurosci. 14, 488-501 (2013).

52. Davis, M. Neural systems involved in fear and anxiety measured with fearpotentiated startle. Am. Psychol. 61, 741-756 (2006).

53. Dong, H. W. \& Swanson, L. W. Projections from bed nuclei of the stria terminalis, posterior division: implications for cerebral hemisphere regulation of defensive and reproductive behaviors. J. Comp. Neurol. 471, 396-433 (2004).

54. Dong, H. W. \& Swanson, L. W. Organization of axonal projections from the anterolateral area of the bed nuclei of the stria terminalis. J. Comp. Neurol. 468, 277-298 (2004).

55. Tovote, P., Fadok, J. P. \& Luthi, A. Neuronal circuits for fear and anxiety. Nat. Rev. Neurosci. 16, 317-331 (2015).

56. Sierra-Mercado, D., Padilla-Coreano, N. \& Quirk, G. J. Dissociable roles of prelimbic and infralimbic cortices, ventral hippocampus, and basolateral amygdala in the expression and extinction of conditioned fear. Neuropsychopharmacology 36, 529-538 (2011).

57. Diehl, M. M. et al. Active avoidance requires inhibitory signaling in the rodent prelimbic prefrontal cortex. eLife 7, https://doi.org/10.7554/eLife.34657 (2018).
58. Vertes, R. P. \& Kocsis, B. Brainstem-diencephalo-septohippocampal systems controlling the theta rhythm of the hippocampus. Neuroscience 81, 893-926 (1997).

59. Buzsaki, G. Theta oscillations in the hippocampus. Neuron 33, 325-340 (2002).

60. Lesting, J. et al. Directional theta coherence in prefrontal cortical to amygdalohippocampal pathways signals fear extinction. PLoS ONE 8, e77707 (2013).

61. Zhang, J. et al. Decreased gray matter volume in the left hippocampus and bilateral calcarine cortex in coal mine flood disaster survivors with recent onset PTSD. Psychiatry Res. 192, 84-90 (2011).

62. Lebow, M. A. \& Chen, A. Overshadowed by the amygdala: the bed nucleus of the stria terminalis emerges as key to psychiatric disorders. Mol. Psychiatry 21, 450-463 (2016).

63. Schelling, G., Roozendaal, B. \& De Quervain, D. J. Can posttraumatic stress disorder be prevented with glucocorticoids? Ann. N. Y Acad. Sci. 1032, 158-166 (2004)

64. Clinton, S. M., Watson, S. J. \& Akil, H. High novelty-seeking rats are resilient to negative physiological effects of the early life stress. Stress 17, 97-107 (2014).

65. King, J. A. et al. Procedure for minimizing stress for fMRI studies in conscious rats. J. Neurosci. Methods 148, 154-160 (2005).

66. Bergmann, E., Zur, G., Bershadsky, G. \& Kahn, I. The Organization of Mouse and Human Cortico-Hippocampal Networks Estimated by Intrinsic Functional Connectivity. Cereb. Cortex 26, 4497-4512 (2016).

67. Yoshida, K. et al. Physiological effects of a habituation procedure for functional MRI in awake mice using a cryogenic radiofrequency probe. $J$. Neurosci. Methods 274, 38-48 (2016).

68. Chang, P. C. et al. Novel method for functional brain imaging in awake minimally restrained rats. J. Neurophysiol. 116, 61-80 (2016).

69. Liang, Z., Liu, X. \& Zhang, N. Dynamic resting state functional connectivity in awake and anesthetized rodents. Neuroimage 104, 89-99 (2015).

70. Power, J. D., Barnes, K. A., Snyder, A. Z., Schlaggar, B. L. \& Petersen, S. E. Spurious but systematic correlations in functional connectivity MRI networks arise from subject motion. Neuroimage 59, 2142-2154 (2012).

\section{Acknowledgements}

The present study was partially supported by National Institute of Neurological Disorders and Stroke Grant R01NS085200 (PI: Nanyin Zhang, Ph.D) and National Institute of Mental Health Grant R01MH098003 and RF1MH114224 (PI: Nanyin Zhang, Ph.D) and R37MH058883 (PI: Gregory Quirk).

\section{Author contributions}

N.Z. designed the research; P.D.P., D.D., and A.V. performed animal experiments; D.D., H.B.R., G.J.Q., and Y.M. performed data analysis; N.Z., D.D., P.D.P., and G.J.Q. wrote the paper.

\section{Additional information}

Supplementary Information accompanies this paper at https://doi.org/10.1038/s41467019-09926-z.

Competing interests: The authors declare no competing interests.

Reprints and permission information is available online at http://npg.nature.com/ reprintsandpermissions/

Journal Peer Review Information: Nature Communications thanks Marcelo Febo, Bruce McEwen, and the other anonymous reviewer(s) for their contribution to the peer review of this work.

Publisher's note: Springer Nature remains neutral with regard to jurisdictional claims in published maps and institutional affiliations.

(c) (i) Open Access This article is licensed under a Creative Commons (c) Attribution 4.0 International License, which permits use, sharing, adaptation, distribution and reproduction in any medium or format, as long as you give appropriate credit to the original author(s) and the source, provide a link to the Creative Commons license, and indicate if changes were made. The images or other third party material in this article are included in the article's Creative Commons license, unless indicated otherwise in a credit line to the material. If material is not included in the article's Creative Commons license and your intended use is not permitted by statutory regulation or exceeds the permitted use, you will need to obtain permission directly from the copyright holder. To view a copy of this license, visit http://creativecommons.org/ licenses/by/4.0/

() The Author(s) 2019 